Owens, Peter J.; Walther, Hansjoachim:

Hamiltonicity in multitriangular graphs

URN: $\quad$ urn:nbn:de:gbv:ilm1-2020200016

\begin{tabular}{ll}
\hline Original published in: & $\begin{array}{l}\text { Discussiones mathematicae. Graph theory / Uniwersytet Zielonogórski, } \\
\text { Wydział Matematyki, Informatyki i Ekonometrii. - Warsaw : De Gruyter } \\
\end{array}$ \\
& Open. - 15 (1995), 1, p. 77-88. \\
& 1995 \\
Original published: & $2083-5892$ \\
ISSN: & $\begin{array}{l}10.7151 / \text { dmgt.1009 } \\
\text { DOI: }\end{array}$ \\
[Visited: & This work is licensed under a Creative Commons Attribution- \\
& $\begin{array}{l}\text { NonCommercial-NoDerivatives 3.0 Unported license. } \\
\text { To view a copy of this license, visit } \\
\text { http://creativecommons.org/licenses/BY-NC-ND/3.0/ }\end{array}$ \\
\hline
\end{tabular}




\title{
HAMILTONICITY IN MULTITRIANGULAR GRAPHS
}

\author{
Peter J. OWEns \\ University of Surrey Guildford, GU2 5XH, United Kingdom \\ and \\ HANSJOACHIM WALTHER \\ Technical University Ilmenau, Institite of Mathematies \\ PF 327, D-98684 Ilmenau, Germany
}

\begin{abstract}
The family of 5 -valent polyhedral graphs whose faces are all triangles or $3 s$-gons, $s \geq 9$, is shown to contain non-hamiltonian graphs and to have a shortness exponent smaller than one.
\end{abstract}

Keywords: polyhedral graphs, longest cycles, shortness exponent

1991 Mathematics Subject Classification: 05C38, 05C45

\section{INTRODUCTION}

All graphs in this paper are simple, that is, they have no loops or multiple edges. For a graph $\boldsymbol{G}=(V, E)$, let $v(\boldsymbol{G})=|V(\boldsymbol{G})|$ be the number of vertices and $c(\boldsymbol{G})$ be the length of a maximum cycle. Thus $\boldsymbol{G}$ is non-hamiltonian if $c(\boldsymbol{G})<v(\boldsymbol{G})$. For a family $\mathcal{G}$ of graphs the shortness coefficient, $\rho(\mathcal{G})$, and shortness exponent, $\sigma(\mathcal{G})$, are defined as in Grünbaum and Walther [2] by $\rho(\mathcal{G})=\liminf _{\boldsymbol{G} \in \mathcal{G}} \frac{c(\boldsymbol{G})}{v(\boldsymbol{G})}, \quad \sigma(\mathcal{G})=\liminf _{\boldsymbol{G} \in \mathcal{G}} \frac{\log c(\boldsymbol{G})}{\log v(\boldsymbol{G})}$.

We consider polyhedral graphs, that is, 3-connected planar graphs. The first example of a non-hamiltonian 3-regular polyhedral graph was given by Tutte [12]. More recently Holton and McKay [4] showed that the smallest such graphs have 38 vertices. Walther [13] showed how to convert a nonhamiltonian 3-regular polyhedral graph into non-hamiltonian 4-regular and 5-regular graphs.

The theorem of Grinberg [1] made it easy to construct non-hamiltonian polyhedral graphs. Grinberg gave as an example a graph in which, apart from one face, all faces are 5 -gons or 8 -gons. This perhaps suggested the 
search for non-hamiltonian regular polyhedral graphs with only two types of faces.

Let $\boldsymbol{\Gamma}(r ; p, q)$ be the family of all $r$-regular polyhedral graphs whose faces are only $p$-gons or $q$-gons, $p<q$. It follows from Euler's relation for polyhedra that $\boldsymbol{\Gamma}(r ; p, q)$ is empty unless $(r, p) \in\{(3,5),(3,4),(3,3),(4,3)$, $(5,3)\}$. Moreover, the families $\boldsymbol{\Gamma}(3 ; 3,4), \boldsymbol{\Gamma}(3 ; 3,5)$ and $\boldsymbol{\Gamma}(3 ; 4,5)$ are finite and contain only hamiltonian graphs, while $\boldsymbol{\Gamma}(3 ; 3, q)$ is empty for $q \geq 10$. In other cases $\boldsymbol{\Gamma}(r ; p, q)$ is infinite.

In Zaks [16] non-hamiltonian graphs in $\boldsymbol{\Gamma}(3 ; 5,8)$ are constructed. A number of later papers, including $[3,5,6,7,8,9,10,11,14,17,18]$, give non-hamiltonian graphs in other families $\boldsymbol{\Gamma}(r ; p, q)$ and inequalities involving shortness parameters. Most such results are of the form $\rho \leq \rho_{0}<1$ (or $\sigma \leq \sigma_{0}<1$ ) for all $q \geq q_{0}$ but in a few cases there are other restrictions on $q$. No non-hamiltonian graphs are known in $\boldsymbol{\Gamma}(3 ; 4, q)$ when $q$ is even and, indeed, none exist if Barnette's conjecture, that every 3-regular bipartite polyhedral graph is hamiltonian, is true.

The inequality $\sigma(\boldsymbol{\Gamma}(3 ; 5, q))<1$ was obtained in Harant [3] for $q \geq$ $83, q \not \equiv 0(\bmod 5)$. In Owens $[8]$ this inequality was shown to hold for $q \geq$ $12, q \not \equiv 0(\bmod 5)$ and, by a different construction, for $q \geq 40, q \equiv 0(\bmod 5)$.

In Owens [6] it was shown that $\sigma(\boldsymbol{\Gamma}(5 ; 3, q))<1$ for $q \geq 14, q \not \equiv$ $0(\bmod 3)$. When $q \equiv 0(\bmod 3)$ the family $\boldsymbol{\Gamma}(5 ; 3, q)$ is a subfamily of the family of all multitriangular graphs, that is, graphs such that the number of edges of every face is divisible by three. Walther [15] showed that the family of 5-regular multitriangular polyhedral graphs has a non-hamiltonian member and even has shortness exponent less than one. He asked how few types of faces there could be for these results to hold. Here, we give best possible answers to these questions by proving the

Theorem. $\sigma(\boldsymbol{\Gamma}(5 ; 3, q))<1$ for $q \geq 27, q \equiv 0(\bmod 3)$.

This result complements [6, Theorem 4]. The construction used is similar to that in [15] but made more general by the use of a technique, called splitting edges, which will now be described.

\section{Constructions and Proofs}

The operation of splitting an edge converts one 5-regular multitriangular polyhedral graph into another. Figure 1 shows three ways to split an edge $A A^{\prime}$ of a 5 -regular graph. 

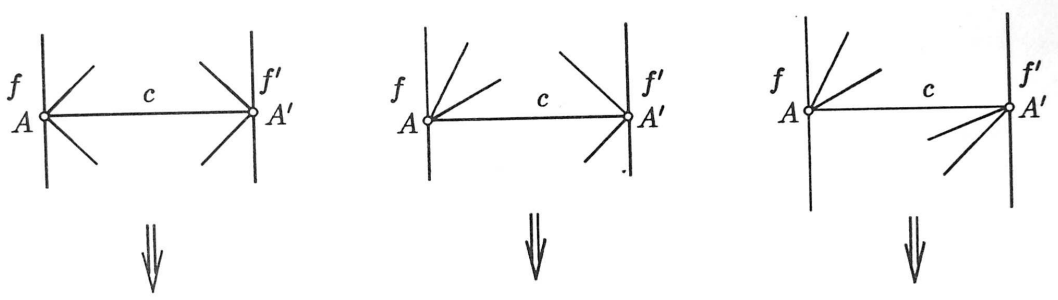

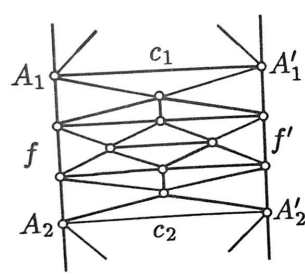

(a)

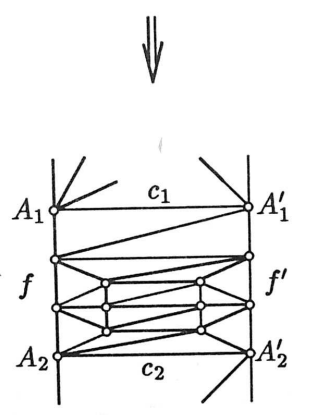

(b)

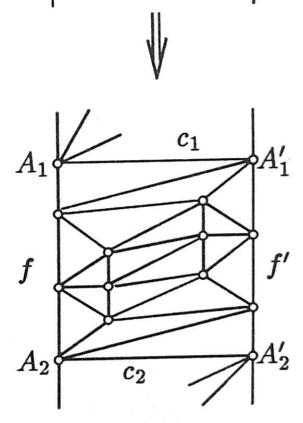

(c)

Figure 1

The edge $A A^{\prime}$ is replaced by two edges $A_{1} A_{1}^{\prime}, A_{2} A_{2}^{\prime}$ and eighteen new triangular faces are produced. Also, two faces $f, f^{\prime}$ incident at $A A^{\prime}$ are enlarged by three edges each. It is essential to specify which faces are to be enlarged because different choices are possible (see Figure 1). After an edge $A A^{\prime}$ has been split the operation can be repeated by splitting $A_{1} A_{1}^{\prime}$ or $A_{2} A_{2}^{\prime}$, with the same (or different) choice of faces $f, f^{\prime}$. Evidently splitting an edge preserves the properties of being polyhedral, multitriangular and 5-regular.

Let $q=3 s$. A graph that can be a subgraph of a 5 -regular multitriangular polyhedral graph is shown in Figure 2(a). It is 5-regular apart from the three vertices $X_{1}, Y_{1}, Z_{1}$, which we call its linking vertices. 


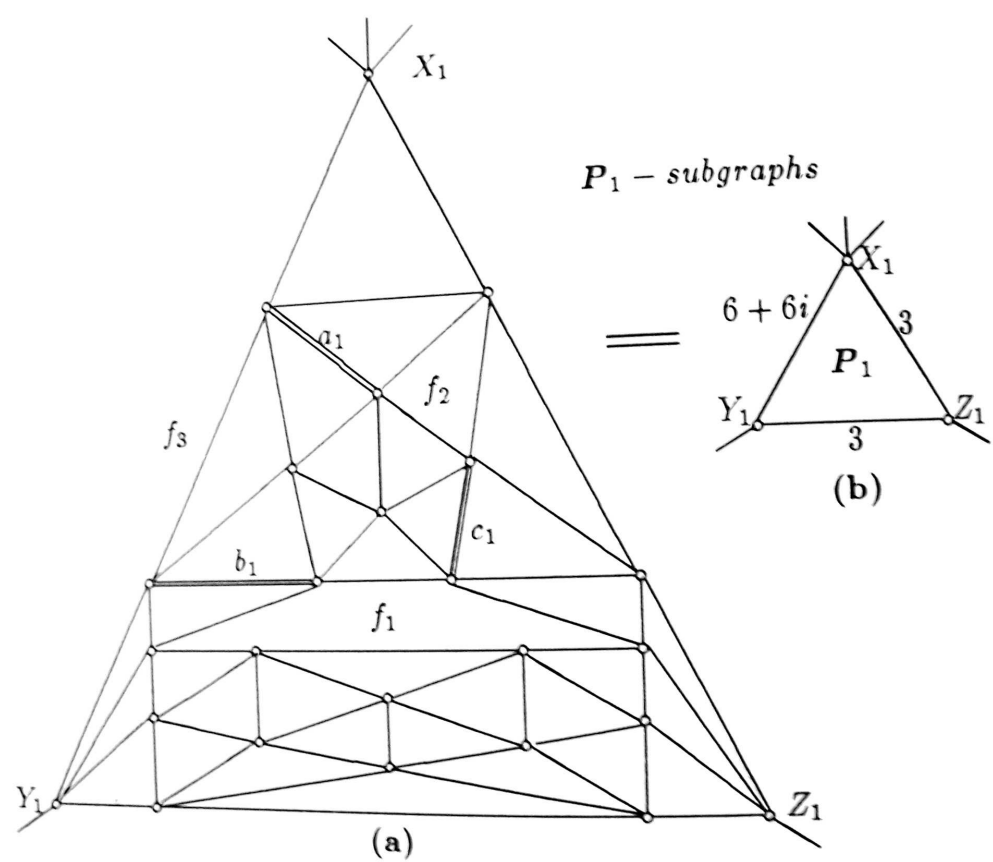

Figure 2

The half edges incident at the linking vertices show how the subgraph is attached to the rest of a 5 -regular polyhedral graph in which it occurs. Let the edges $a_{1}, b_{1}, c_{1}$ shown as double lines in Figure 2(a) be split $1+i, i, s-$ $i-2$ times respectively in such a way that the only faces to be enlarged are $f_{1}, f_{2}$ and $f_{3}$. It is easy to check that $f_{1}$ and $f_{2}$ become $3 s$-gons and $f_{3}$ gains $3+6 i$ extra edges. We denote the new subgraph by $\boldsymbol{P}_{1}(3,6+6 i, 3 ; 3 s)$ and represent it by a labelled triangle, as shown in Figure 2(b). The numbers outside the triangle show how many edges this $\boldsymbol{P}_{1}$-subgraph supplies to the three adjoining faces of a graph in which it occurs. If that graph is in $\boldsymbol{\Gamma}(5 ; 3,3 s)$, then $6+6 i<3 s$. This implies that $s-i-2$ is non-negative, which is essential since it is the number of times $c_{1}$ has been split.

In a similar way a subgraph $\boldsymbol{P}_{2}(1+3 j+6 k, 2+3 j, 2 ; 3 s)$ is obtained from the subgraph shown in Figure 3 (a) by splitting the edges shown as double lines. 

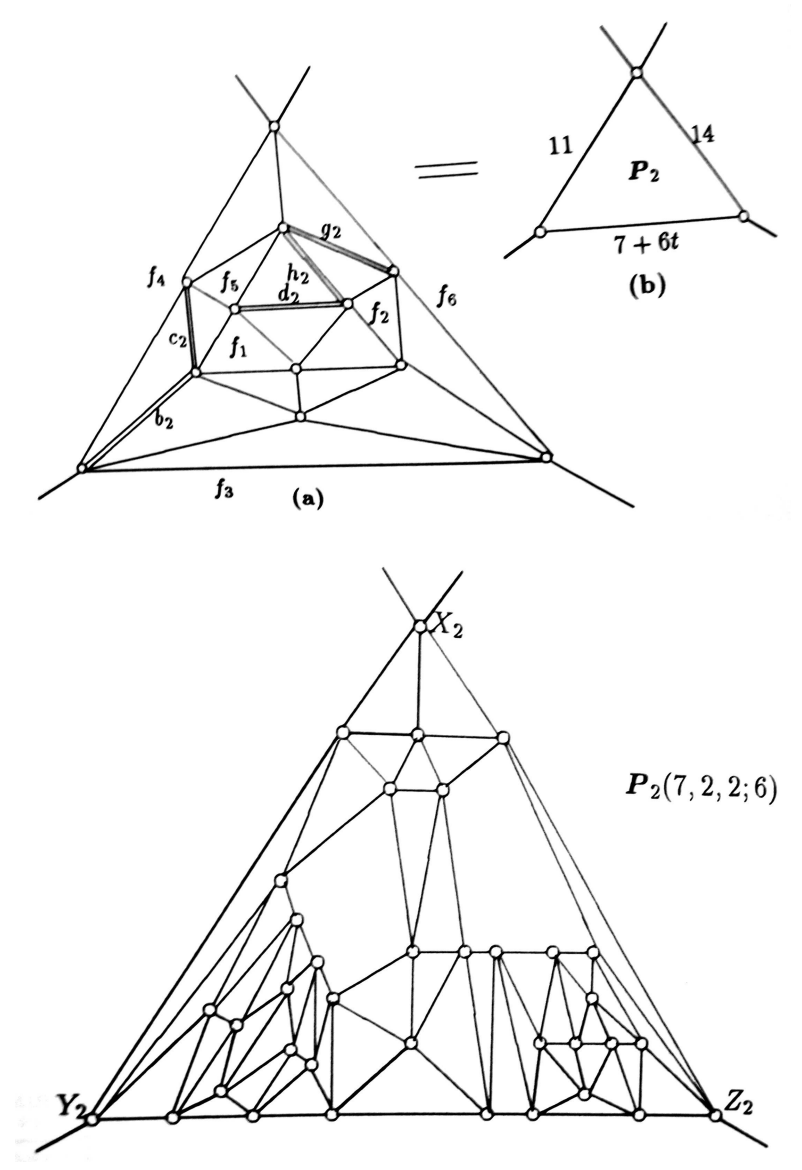

Figure 3

In fact, let the edges $a_{2}, b_{2}, c_{2}, d_{2}$ be split $j+k, k, j, s-j-k-1$ times in such a way that the pairs of faces which are enlarged are $f_{2}$ and $f_{3}, f_{1}$ and $f_{3}, f_{1}$ and $f_{4}, f_{1}$ and $f_{2}$, respectively. The faces $f_{1}$ and $f_{2}$ become $3 s$-gons and the numbers of edges supplied by the $\boldsymbol{P}_{2}$-subgraph to the adjoining faces are as shown in Figure 3(b). In Figure 3(c) we show $\boldsymbol{P}_{2}(7,2,2 ; 6)$ as an example of the construction for $j=0, k=1$. 
$\mathbf{R}(3,5+3 j, 1+3 j+6 k, 8+6 i ; q) \quad \mathbf{R}(3,5,1+3 j+6 k, 8+6 i+3 j ; q)$
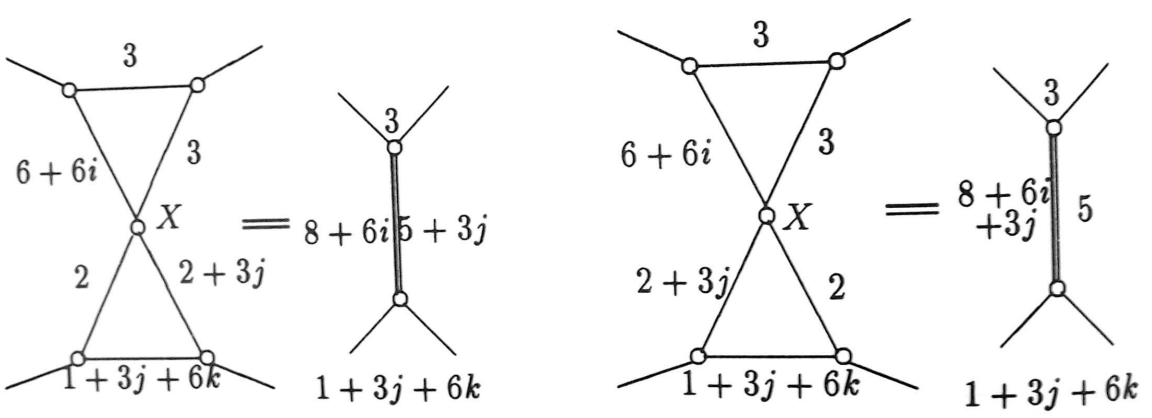

Figure 4

A subgraph with four linking vertices can now be obtained by identifying the vertex $X_{1}$ of a $\boldsymbol{P}_{1}$-subgraph with the vertex $X_{2}$ of a $\boldsymbol{P}_{2}$-subgraph to form a vertex $X$. This can be done in two ways, as shown in Figure 4, and we denote the two sorts of $\boldsymbol{R}$-subgraph by

(a) $\boldsymbol{R}(3,5+3 j, 1+3 j+6 k, 8+6 i ; 3 s)$ and

(b) $\boldsymbol{R}(3,5,1+3 j+6 k, 8+6 i+3 j ; 3 s)$.

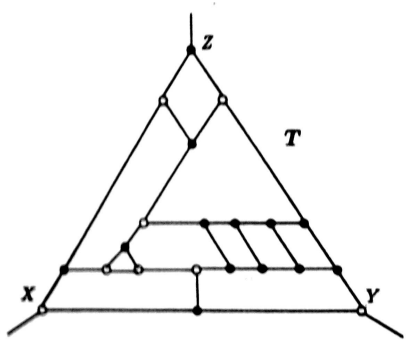

(a)

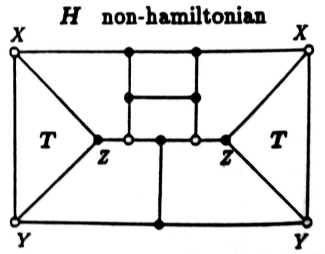

(b)

Figure 5

Figure 5 shows a non-hamiltonian 3-regular polyhedral graph with 50 vertices. 
The subgraph $\boldsymbol{T}$, two copies of which appear in , is obtained from the usual Tutte subgraph by replacing one 4-gon by three 4-gons and one vertex by a triangle.

We state without proof a property of $\boldsymbol{T}$ that follows easily from the corresponding property of the Tutte subgraph:

Every path from $X$ to $Y$ in $\boldsymbol{T}$ misses at least one of the 13 black vertices.

(The idea of black vertices was used in [2] and corresponds to that of $z$-vertices in [6].) Hence every cycle in misses at least one of the 32 black vertices.

The next step is to convert, with the aid of $\boldsymbol{R}$-subgraphs, into a nonhamiltonian graph in $\boldsymbol{\Gamma}(5 ; 3,3 s)$.

Two cases must be considered, according to the parity of $s$.

Case 1. (Odd $s)$ Let $s=9+2 t, q=27+6 t$, where $t \geq 0$.

The doubled lines in Figure 6 indicate the edges of a 1 -factor of . Replace each edge of this 1 -factor by an $\boldsymbol{R}$-subgraph. The numbers placed round the doubled lines, taken in conjunction with Figure 4, show which particular $\boldsymbol{R}$-subgraphs are to be used. In Figure $6,+$ is an abbreviation for $+6 t$. It is easy to verify that there are values of $i, j$ and $k$ that produce all the required $\boldsymbol{R}$-subgraphs. Moreover, all faces of the new graph $\boldsymbol{G}$ that are shown explicitly are $(27+6 t)$-gons (implicitly there are triangles, namely inside $\boldsymbol{R}$-subgraphs). Hence $\boldsymbol{G} \in \boldsymbol{\Gamma}(5 ; 3, q)$.

Let $C$ be any cycle in $\boldsymbol{G}$. Under the transformation that converts $\boldsymbol{G}$ back into, $C$ becomes a cycle $C^{\prime}$ in, or possibly a single vertex. If $C$ contains an edge of a particular $\boldsymbol{P}_{1^{-}}$or $\boldsymbol{P}_{2}$-subgraph, then $C^{\prime}$ contains the vertex of corresponding to this subgraph of $\boldsymbol{G}$. Since $C^{\prime}$ must miss a black vertex of , $C$ must miss all the edges of the corresponding $\boldsymbol{P}_{1-}$ or $\boldsymbol{P}_{2}$-subgraph of $\boldsymbol{G}$.

Case 2. (Even $s$ ) Let $s=10+2 t, q=30+6 t$, where $t \geq 0$.

The graph $\boldsymbol{G}$ constructed in Case 1 has an odd number of $q$-gons, including four within each $\boldsymbol{R}$-subgraph. Since two faces are enlarged (by three edges each) whenever an edge is split, it is not possible by splitting edges to replace all $q$-gons by $(q+3)$-gons and not alter any other faces.

Figure 7 shows a construction for even $s$ similar to that given in Figure 6 for odd $s$. One (but only one) of the required $\boldsymbol{R}$-subgraphs, namely $\boldsymbol{R}(3,17,7+6 t, 17+6 t ; q)$, cannot be obtained as in Figure 4 . To obtain this extra subgraph, take the subgraph shown in Figure 8(a) and let the edges $b_{2}, c_{2}, d_{2}, g_{2}, h_{2}$ be split $2+2 t, 3,4,4,5+2 t$ times in such a way 


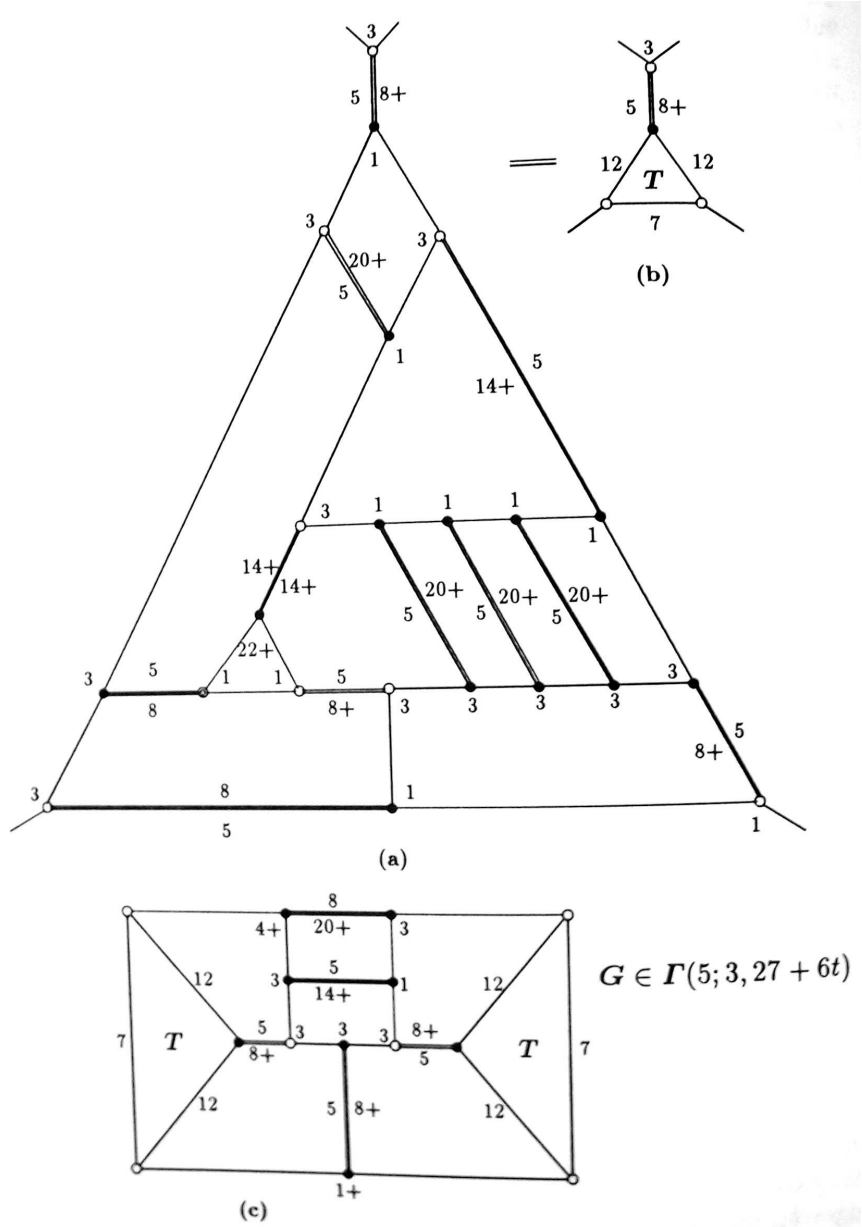

Figure 6 


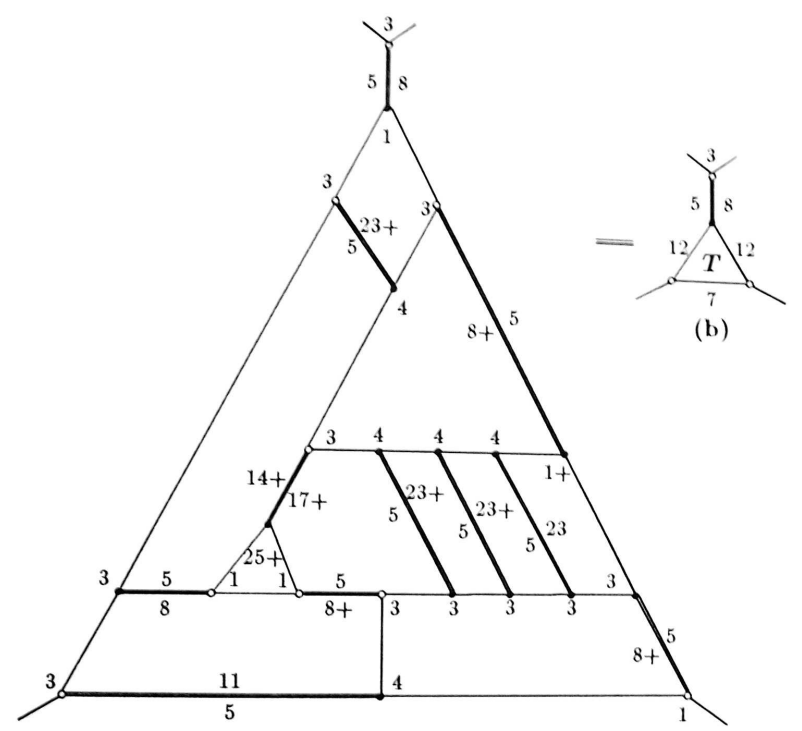

(a)

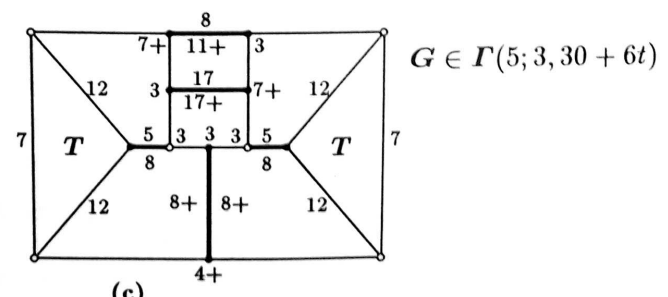

Figure 7 


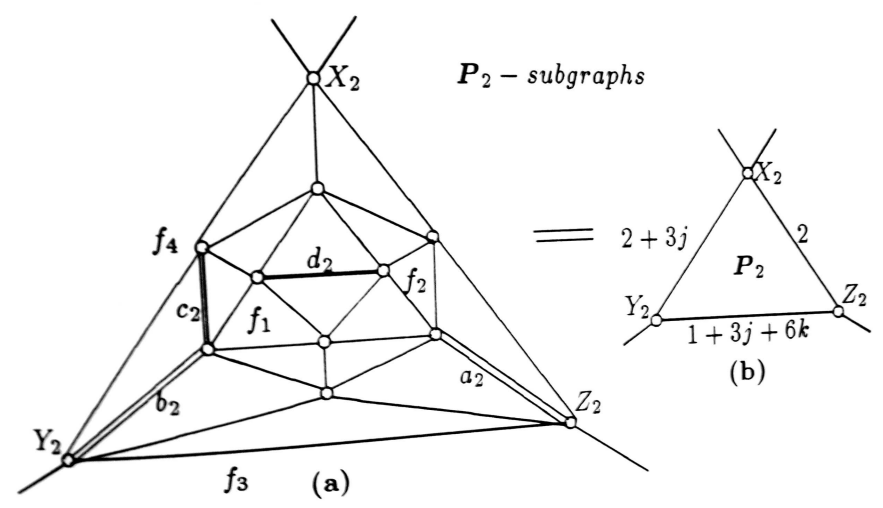

Figure 8

that the pairs of faces enlarged are $f_{1}$ and $f_{3}, f_{1}$ and $f_{4}, f_{1}$ and $f_{2}, f_{5}$ and $f_{6}, f_{2}$ and $f_{5}$, respectively. The interior faces $f_{1}, f_{2}, f_{5}$ become $q$-gons and the new $\boldsymbol{P}_{2}$-subgraph supplies $7+6 t, 11,14$ edges to the three adjoining faces of a graph in which it occurs. By joining this $\boldsymbol{P}_{2}$-subgraph to $\boldsymbol{P}_{1}(3,6+6 t, 3 ; q)$ we obtain the required subgraph $\boldsymbol{R}(3,17,7+6 t, 17+6 t ; q)$. This completes Case 2 . We now have a nonhamiltonian graph $\boldsymbol{G} \in \boldsymbol{\Gamma}(5 ; 3, q)$, for all $q \geq 27$ such that $q \equiv 0(\bmod 3)$.

A routine argument, using [6, Lemma 2], leads to the inequality

$$
\sigma(\boldsymbol{\Gamma}(5 ; 3, q)) \leq \frac{\log 30}{\log 31}
$$

for $q \geq 27, q \equiv 0(\bmod 3)$.

\section{REMARKS}

(1) As far as we are aware, it is not known whether there exist nonhamiltonian graphs in any of the following families:

$\boldsymbol{\Gamma}(3 ; 5,6)$;

$\boldsymbol{\Gamma}(3 ; 4, q)$, where $q$ is even and $q \geq 8$;

$\boldsymbol{\Gamma}(4 ; 3, q)$, where $4 \leq q \leq 11$;

$\boldsymbol{\Gamma}(5 ; 3, q)$, where $4 \leq q \leq 13$ or $q=15,18,21,24$. 
(2) A theorem of Tutte implies that every non-hamiltonian polyhedral graph $\boldsymbol{G}$ has connectivity exactly three. If $\boldsymbol{G}$ is regular and 5 -valent, then its cyclic edge-connectivity is at most six [13, p.295]. This maximum is attained by the non-hamiltonian 5-valent graphs constructed in [6] but not by those in the present paper, because only four edges join an $\boldsymbol{R}$-subgraph to the rest of a graph in which it appears. We therefore ask whether there is, for some $k$, a cyclically 6 -edge-connected non-hamiltonian graph in $\boldsymbol{\Gamma}(5 ; 3,3 k)$.

\section{REFERENCES}

[1] E. J. Grinberg, Planehomogeneous graphs of degree three without Hamiltonian circuits, Latvian Math. Yearbook 4 (1968) 51-58 (in Russian).

[2] B. Grünbaum and H. Walther, Shortness exponents of families of graphs, J. Combin. Theory (A) 14 (1973) 364-385.

[3] J. Harant, Über den Shortness Exponent regulärer Polyedergraphen mit genau zwei Typen von Elementarflächen, Thesis A, Ilmenau Institute of Technology (1982).

[4] D. A. Holton and B. D. McKay, The smallest non-hamiltonian 3-connected cubic planar graphs have 38 vertices, J. Combin. Theory (B) 45 (1988) 305319, with correction, J. Combin. Theory (B) 47 (1989) 248.

[5] P. J. Owens, Non-hamiltonian simple 3-polytopes whose faces are all 5-gons or 7-gons, Discrete Math. 33 (1981) 107-109.

[6] P. J. Owens, Regular planar graphs with faces of only two types and shortness parameters, J. Graph Theory 8 (1984) 253-275.

[7] P. J. Owens, Simple 3-polytopal graphs with edges of only two types and shortness coefficients, Discrete Math. 59 (1986) 107-114.

[8] P. J. Owens, Shortness exponents, simple polyhedral graphs and large bridges, unpublished.

[9] M. Tkáč, Shortness coefficients of simple 3-polytopal graphs with edges of only two types, Discrete Math. 103 (1992) 103-110.

[10] M. Tkáč, Simple 3-polytopal graphs with edges of only two types and shortness coefficients, Math. Slovaca 42 (1992) 147-152.

[11] M. Tkáč, On shortness coefficients of simple 3-polytopal graphs with edges of only one type of face besides triangles, Discrete Math. 128 (1994) 407-413.

[12] W. T. Tutte, On Hamiltonian circuits, J. London Math. Soc. 21 (1946) 98-101.

[13] H. Walther, Über das Problem der Existenz von Hamiltonkreisen in planaren regulären Graphen, Math. Nachr. 39 (1969) 277-296. 
[14] H. Walther, Note on the problems of J.Zaks concerning Hamiltonian 3polytopes, Discrete Math. 33 (1981) 107-109.

[15] H. Walther, A non-hamiltonian five-regular multitriangular polyhedral graph (to appear).

[16] J. Zaks, Non-Hamiltonian non-Grinbergian graphs, Discrete Math. 17 (1977) 317-321.

[17] J. Zaks, Non-Hamiltonian simple 3-polytopes having just two types of faces, Discrete Math. 29 (1980) 87-101.

[18] J. Zaks, Non-hamiltonian simple planar graphs, in: Annals of Discrete Math. 12 (North - Holland, 1982) 255-263.

Received 19 October 1994 